\title{
Treatment of Temporomandibular Joint (TMJ) Reankylosis with Preservation of the TMJ Prosthesis and Use of Fat Graft: A Case Report and Review of the Literature
}

\author{
Risimati Ephraim Rikhotso, Mohammed Bobat \\ Department of Maxillofacial and Oral Surgery, University of the Witwatersrand, Johannesburg, South Africa \\ Email: erikhotso@gmail.com
}

How to cite this paper: Rikhotso, R.E. and Bobat, M. (2018) Treatment of Temporomandibular Joint (TMJ) Reankylosis with Preservation of the TMJ Prosthesis and Use of Fat Graft: A Case Report and Review of the Literature. Open Journal of Stomatolo$g y, 8,71-80$.

https://doi.org/10.4236/ojst.2018.83006

Received: February 14, 2018

Accepted: March 11, 2018

Published: March 14, 2018

Copyright $\odot 2018$ by authors and Scientific Research Publishing Inc. This work is licensed under the Creative Commons Attribution International License (CC BY 4.0).

http://creativecommons.org/licenses/by/4.0/

\begin{abstract}
We report on a case of TMJ reankylosis (heterotopic bone) following total TMJ alloplastic reconstruction in a 40-year-old Black male. The heterotopic bone was excised following removal of the fossa component. The fossa component was re-inserted, and the periarticular tissue was grafted with fat procured from the abdomen. Patient was placed on an aggressive physiotherapy programme which was monitored for a period of 6 months. He returned for review one year later with a mouth opening of $27 \mathrm{~mm}$. We present also a short review of the literature on heterotopic bone formation. This case is of particular interest in that despite the heterotopic bone around it; the prosthesis was preserved after the bone was removed.
\end{abstract}

\section{Keywords}

TMJ Reankylosis, Heterotopic Bone, Alloplasts, Fat Graft

\section{Introduction}

Temporomandibular joint (TMJ) ankylosis is defined as the inappropriate fusion of the mandibular condyle to the mandibular fossa and its surrounding bone. This fusion may be bony, fibrous or fibro-osseous in nature [1]. The most commoncause is traumatic injury to the TMJ complex followed by local infection with rheumatoid arthritis and its related conditions as potential but less likely causes [2]. The exact pathogenesis of TMJ ankylosis in traumatic injuries is unclear; however it has been suggested that joint hypomobility, young patient age as well as the morphology of the condylar fracture may increase the risk of an- 
kyloses [3].

The goals of treatment are to remove the ankylotic mass as well as improve the mandibular range of motion and masticatory function. Treatment options include bony autografts (costochondral, clavicular), interpositional grafts, alloplastic TMJ prostheses as well as gap arthroplasty [4] [5].

Alloplastic TMJ prosthesis provide significant benefits such as lack of donor site morbidity, dimensional stability compared to autogenous costochondral grafts and the ability to provide earlier return to function as well as earlier initiation of post-operative physiotherapy [6]. They also reduce the risk of occlusal disturbances associated with gap and interpositional arthroplasties [4]. Disadvantages of TMJ prostheses include cost and judicious use in a skeletally immature patient due to predicted future craniofacial growth [6]. Complications associated with alloplastic prosthesis include infection, screw loosening, as well as heterotopic bone formation [4] [5] [6].

We report on the management of a case which developed heterotopic bone formation around TMJ prosthesis as well as a discussion of the available literature relating to this complication.

\section{Case Report}

A 40 year old Black male patient presented to the Chris Hani Baragwanath Hospital Maxillofacial unit with the main complaint that he was unable to open his mouth and chew. Surgical history was significant for bilateral total alloplastic TMJ reconstruction done two years previously (Biomet Total TMJ prostheses, Biomet Microfixation, Jacksonville, FL, USA) post-traumatic ankylosis. This was followed by aggressive post-operative physiotherapy for a period of 3 months. The patient was followed up for six months, after which he was lost to follow-up

CT SCANS evaluation revealed significant heterotopic bone formation on the medial aspect of the left TMJ condylar component to the base of skull and extending onto the fossa component laterally (Figure 1(a) and Figure 1(b)).

The left TMJ was approached via a hockey-stick preauricular incision. The fossa component (Figure 2(a) and Figure 2(b)) was carefully unscrewed and removed to expose the head of the condylar prosthetic component surrounded by bone (Figure 3). The heterotopic bony elements were carefully removed with no attempt to remove the condylar prosthetic component, creating a gap of approximately $1,5-2 \mathrm{~cm}$ from the head of the condylar prosthesis to the cranial base (Figure 4). The fossa component was then re-inserted and the mandible was stretched to approximately $45 \mathrm{~mm}$ interincisal distance (Figure 5). Autogenous fat graft harvested from the abdomen was packed around the articulating areas of the prosthesis (Figure 6). A negative pressure suction device was inserted and wound closure was performed. The post-operative course was uneventful. Aggressive physiotherapy was reinitiated 1 week after surgery. The patient was closely monitored, especially with regards to physiotherapy, which was constantly supervised for the first 6 months. Follow-up visits were then 


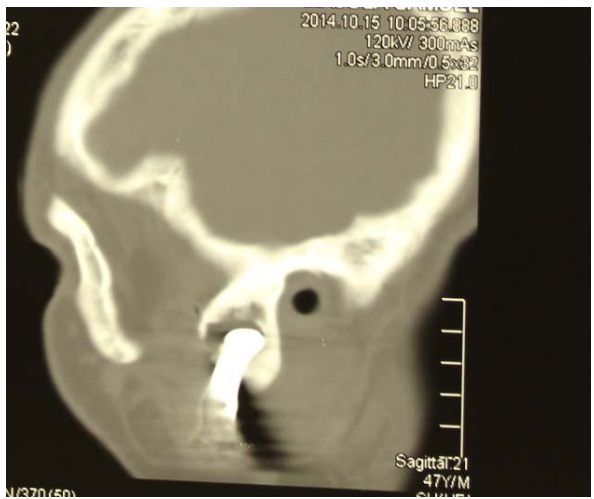

(a)

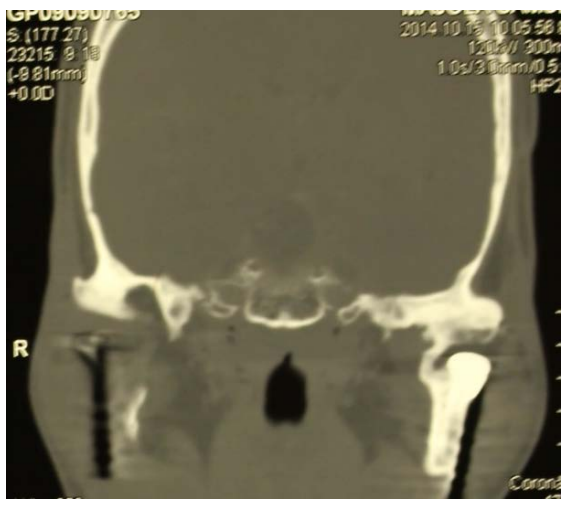

(b)

Figure 1. (a) Coronal CT SCANS showing bony bridge medial to the condylar head prosthesis. (b) Sagittal view showing condylar head prosthesis encased in bone.

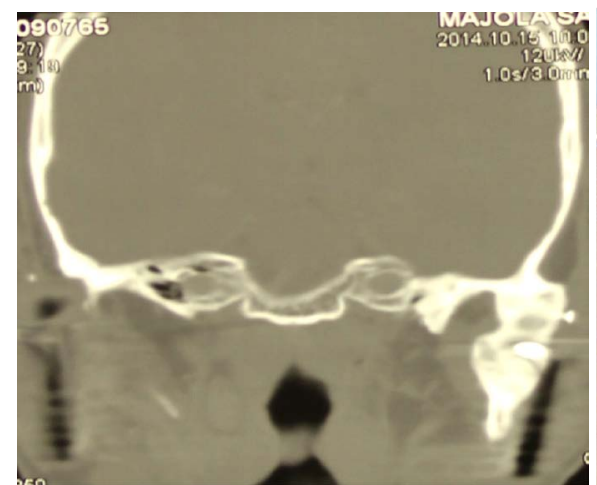

(a)

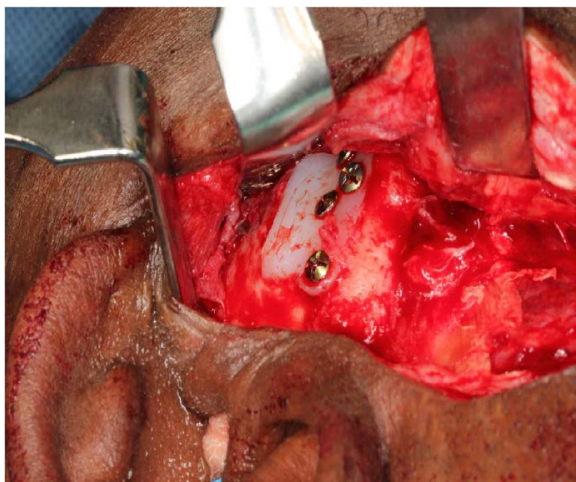

(b)

Figure 2. (a) Condylar head prosthesis surrounded by bone and one of the screws (arrow) holding the fossa component. (b) Illustration of bone (arrow) below the fossa componet prior to its removal.

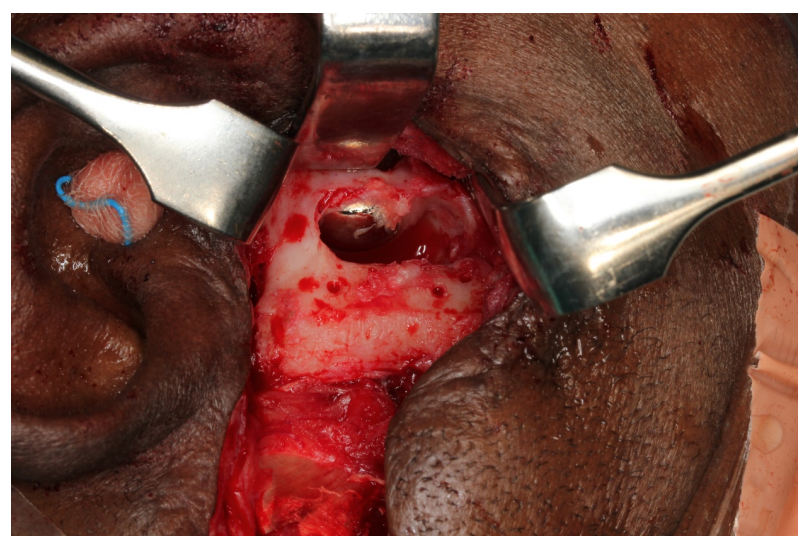

Figure 3. Heterotopic bone encasing condylar head (arrow) prosthesis (post-removal of the fossa component).

conducted at three month intervals. Patient returned for a three year review with an interincisal mouth opening of $27 \mathrm{~mm}$ (Figure 7), with no clinical nor radiographic signs of re-ankylosis. 


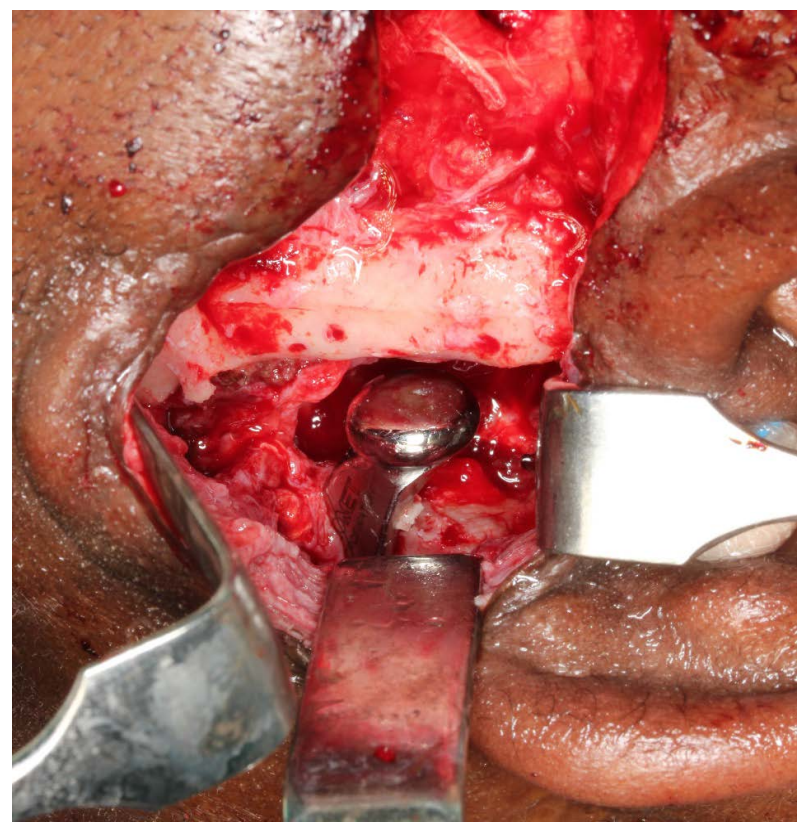

Figure 4. Heterotopic bone excised around the condylar head prosthesis.

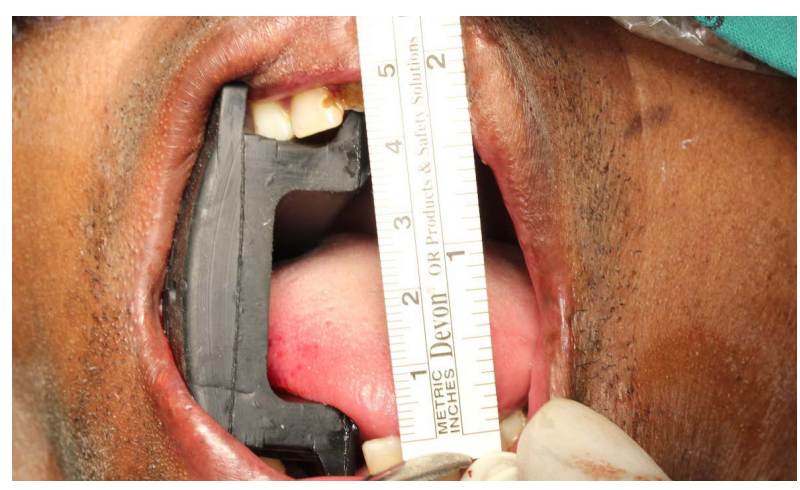

Figure 5. Patient stretched to an inter-incisal mouth opening of $45 \mathrm{~mm}$.

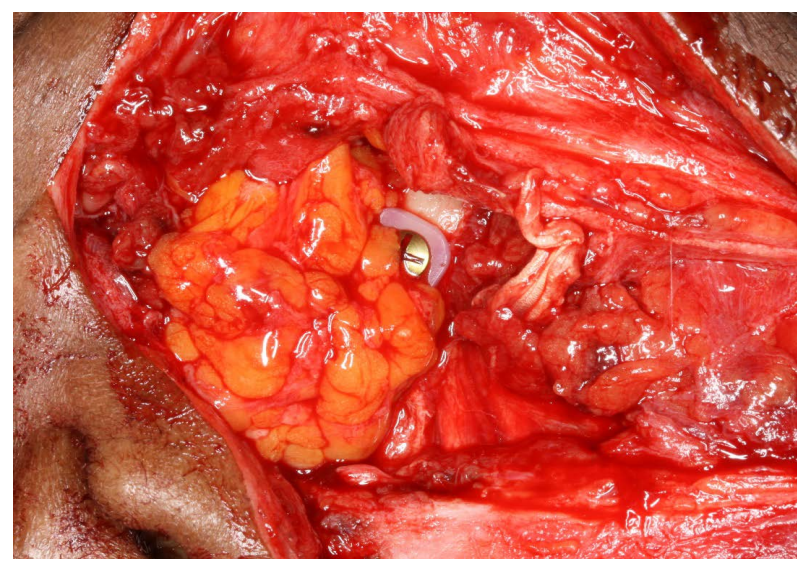

Figure 6. Fat graft packed around the articulating surfaces of the prosthesis. 


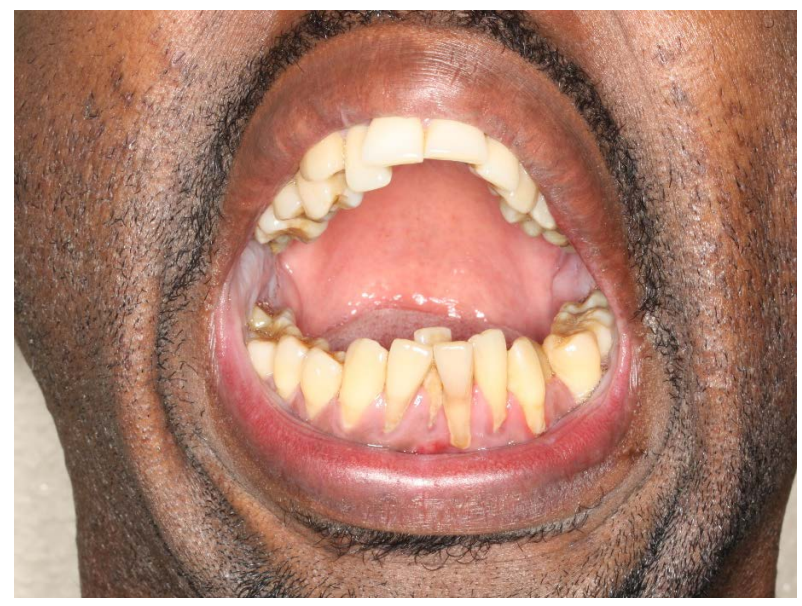

Figure 7. Inter-incisal mouth-opening at a 3-year review.

\section{Discussion}

The use of total alloplastic TMJ prostheses in the management of TMJ ankylosis has eliminated the problems of donor site morbidity and unpredictable growth associated with costochondral grafts. They are however not without complications. Giannakopoulous et al. [7] reported on 442 Biomet joint prostheses over a 3 year period with the loss of 14 prosthetic components (3.2\%) due to infection or heterotopic bone formation. Sanovich et al. [6] reported heterotopic bone formation in 2 out of 106 Biomet joint prostheses, a complication rate of $1.8 \%$.

After infection (2.7\%), acquired heterotopic ossification (HO) (1.24\%) is the second most common post-implantation complication associated with alloplastic TMJ replacement. It is postulated that the inflammatory response to injury and surgical woun [8]ding plays a critical role in the development of $\mathrm{HO}$ and requires three components: osteoinductive factors, skeletal progenitor cells and a permissive tissue environment [9]. The induction of cells of mesenchymal origin, present in a permissive environment created in the peri-articular tissues, leads to their transformation into osteogenic cells. This leads to overactivation of the BMP cascade (BMP-2/4 subtype in particular) through activation of the activin type 1 receptor (ACVRT) resulting in abnormal bone formation [9] [10] [11].

The presence of dead space after total joint prosthesis placement leads to blood clot formation with subsequent organization. Pluripotential cells may then migrate into the area and be induced to differentiate into fibroblast and osteoblasts, with deposition of collagen and bone respectively [12].

The HO process recapitulates the cellular and molecular events of endochondral bone formation in embryonic skeletal development and fracture healing [9]. However, these events are temporarily and spatially unsynchronized, resulting in disorganized and non-homogenous HO bone [9] [13].

The development of $\mathrm{HO}$ around total alloplastic temporomandibular joint reconstruction devices was first described in 1992 by Lindqvist [9] [14] [15]. A number of risk factors for the development of $\mathrm{HO}$ have been described based on 
numerous case reports [14]-[20]:

a) Previously operated joints.

b) Infection.

c) Ankylosis.

d) Reankylosis.

e) Ankylosing spondylitis.

f) Revision of failed autogenous or alloplastic TMJR devices.

g) Patients unco-operative with post-implantation physical therapy.

It appears like lack of physiotherapy together with the fact that this was a multiple operated joint were significant contributors to the pathogenesis of $\mathrm{HO}$ (reankylosis) in our patient.

While this complication rate is low and does not prejudice the superior performance of TMJ prostheses, there is no consensus on the prevention and management of heterotopic bone formation around them. Pharmacologic agents (in particular non-steroidal anti-inflammatory drugs indomethacin and etidronate) have been used in orthopaedics as prophylactic and non-surgical management options with varying success [12] [21] [22] [23]. A meta-analysis reviewing randomised controlled trials for the efficacy of non-selective non-steroidal anti-inflammatory drugs (NSAIDS) and cyclo-oxygenase 2 inhibitors demonstrated equal effectiveness in preventing $\mathrm{HO}$ [24]. The proposed mechanism of action of NSAIDs in preventing $\mathrm{HO}$ is their inhibition of inflammatory prostaglandins and potent co-stimulatory molecules for BMPs [9]. Cella et al. reported that $37 \%$ of patients prescribed NSAIDs for $\mathrm{HO}$ prophylaxis stopped taking these drugs, mainly due to their GIT side effects [23].

In orthopaedics and lately in maxillofacial and oral surgery, low dose radiotherapy is regarded as a safe and efficacious method for preventing HO formation in sites such as hip, elbow, knee and TMJ, despite continued debate about its mechanism of action. Common complication associated with its use in the TMJ include parotitis and xerostomia [16] [18] [25] [26].

Bisphosphonates have also been considered for use in the prevention of $\mathrm{HO}$ (through their production of cytotoxic metabolites within osteoclasts and inhibition of tyrosine phosphatase). Despite their relatively mild GIT effects, renal toxicity,flu-like symptoms and their potential to produce medically related osteonecrosis of the jaws (MRONJ) are causes for concern with chronic use [27]. For these reasons we did not consider prescribing bisphosphonates for our patient.

It has also been reported that replacement of the affected TMJ prosthesis with packing of autologous fat around the joint components reduces the risk of re-ankylosis by reducing dead-space available for hematoma formation and subsequent ossification [28] [29] [30]. Fat not only fills the dead space around the TMJR articulating components but also has a haemostatic effect on the surrounding tissues [20]. According to Wolford, fat graft may be inhibitory to heterotopic bone formation as well as isolate any residual reactive tissue from previous alloplastic failure and disease in the periphery of the region, thus mini- 
mizing formation of bone and reactive tissues around the joint components [12]. Wolford and Karras [17] evaluated fat graft in a comparative study of 35 patients fitted with TMJ Concepts patient-fitted TMJ total joint prosthesis. Of the 35 patients, fifteen were grafted with autologous fat harvested from the abdomen and the other 20 had joints fitted without fat grafts (control group). No radiographic or clinical evidence of heterotopic evidence of heterotopic calcification was noticed in any of the patient in the fat group, whereas 7 control patients developed heterotopic bone and reoperation was required. They concluded that autologous fat transplantation is a useful adjunct to prosthetic TMJ reconstruction by minimizing the occurrence of joint fibrosis and heterotopic calcification, consequently providing an improved range of movement and jaw function.

In his evaluation of 11 patients with 13 ankylosed TMJs treated with gap arthroplasty filled with autogenous dermis-fat harvested from the groin, Dimitroulis [31] found that reankylosis occurred in only one patient at the longest follow-up (average 41.5 months). Mercuri et al. [20] also evaluated 20 patients with 33 ankylosed TMJs fitted with TMJ Concepts total joint prosthesis and periarticular autologous fat. At a mean follow-up of 50.4 months, no case of reankylosis was reported, and maximum incisal mouth opening improved from 11.8 $\mathrm{mm}$ to $32.9 \mathrm{~mm}$.

The most common donor site for fat harvesting is the abdomen, however the buttock, thigh, buccal fat pad and breast are other potential donor sites. Complications associated with harvesting of abdominal fat include infection, seroma and abdominal wall injury [8]. Our patient had no complication post-harvesting of the abdominal fat. Previously we have successfully treated TMJ ankylosis with total alloplastic TMJ reconstruction without any fat graft. We therefore consider fat graft as an adjunct therapy, secondary to physiotherapy which we regard as an integral part in the management of TMJ reankylosis and other hypomobility disorders of the TMJ.

We did not consider replacement of the prosthesis since there was adequate function after removal of the ankylotic mass and the components were not mechanically damaged in any way.

\section{Conclusions}

It is our view that the basis of management of heterotopic bone formation around TMJ prostheses should include complete or adequate removal of the heterotopic mass, intraoperative stretching of the TMJ to re-establish mouth opening and close monitoring of post-operative physiotherapy. There is plausible justification for use of medication (NSAIDs and Bisphosphonates), radiation therapy and packing of autologous fat around the prosthesis but there is paucity of prospective randomised case controlled studies that validate their benefit. Replacement of the prosthesis may be considered however the cost implications would have to be considered.

Further case reports as well as larger multi-center studies are required to establish a standardized protocol for the prevention and management of this com- 
plication and may elucidate reasons for its occurrence.

\section{References}

[1] Movahed, R. and Mercuri, L.G. (2015) Management of Temporomandibular Joint Ankylosis. Oral \& Maxillofacial Surgery Clinics of North America, 27, 27-35. https://doi.org/10.1016/j.coms.2014.09.003

[2] Hegab, A.F. (2015) Outcome of Surgical Protocol for Treatment of Temporomandibular Joint Ankylosis Based on the Pathogenesis of Ankylosis and Re-Ankylosis. A Prospective Clinical Study of 14 Patients. Journal of Oral and Maxillofacial Surgery, 73, 2300-2311. https://doi.org/10.1016/j.joms.2015.06.155

[3] Ferretti, C., Bryant, R., Becker, P. and Lawrence, C. (2005) Temporomandibular Joint Morphology Following Posttraumatic Ankylosis in 26 Patients. International Journal of Oral and Maxillofacial Surgery, 34, 376-381.

https://doi.org/10.1016/j.ijom.2004.09.003

[4] Al-Moraissi, E.A., El-Sharkawy, T.M., Mounair, R.M. and El-Ghareeb, T.I. (2015) A Systematic Review and Meta-Analysis of the Clinical Outcomes for Various Surgical Modalities in the Management of Temporomandibular Joint Ankylosis. International Journal of Oral and Maxillofacial Surgery, 44, 470-482. https://doi.org/10.1016/j.ijom.2014.10.017

[5] Kumar, P., Rattan, V. and Rai, S. (2015) Do Costochondral Grafts Have Any Growth Potential in Temporomandibular Joint Surgery? A Systematic Review. Journal of Oral Biology and Craniofacial Research, 5, 198-202. https://doi.org/10.1016/j.jobcr.2015.06.007

[6] Sanovich, R., Mehta, U., Abramowicz, S., Widmer, C. and Dolwick, M.F. (2014) Total Alloplastic Temporomandibular Joint Reconstruction Using Biomet Stock Prostheses: The University of Florida Experience. International Journal of Oral and Maxillofacial Surgery, 43, 1091-1095. https://doi.org/10.1016/j.ijom.2014.04.008

[7] Giannakopoulos, H.E., Sinn, D.P. and Quinn, P.D. (2012) Biomet Microfixation Temporomandibular Joint Replacement System: A 3-Year Follow-Up Study of Patients Treated during 1995 to 2005. Journal of Oral and Maxillofacial Surgery, 70, 787-794. https://doi.org/10.1016/j.joms.2011.09.031

[8] TMJ Concepts, Ventura, CA, USA. Personal Communication, 2016.

[9] Mercuri, L.G. and Saltzman, B.M. (2017) Acquired Heterotopic Ossification of the Temporomandibular Joint. International Journal of Oral and Maxillofacial Surgery. https://doi.org/10.1016/j.ijom.2017.06.016

[10] Shore, E.M. and Kaplan, F.S. (2010) Inherited Human Diseases of Heterotopic Ossification. Nature Reviews Rheumatology, 6, 1281-1285.

https://doi.org/10.1038/nrrheum.2010.122

[11] Ranganathan, K., Loder, S., Agarwal, S., et al. (2015) Heterotopic Ossification: Basic Science Principles and Clinical Correlates. The Journal of Bone and Joint Surgery. American Volume, 97, 1101-1111. https://doi.org/10.2106/JBJS.N.01056

[12] Wolford, L., Movahed, R., Teschke, M., Fimmers, R., Havard, D. and Shneiderman, E. (2016) Temporomandibular Joint Ankylosis Can Be Successfully Treated with TMJ Concepts Patient-Fitted Total Joint Prosthesis and Autogenous Fat Graft. Journal of Oral and Maxillofacial Surgery, 74, 1215-1227. https://doi.org/10.1016/j.joms.2016.01.017

[13] Yang, N., Reginato, A.M. and Medici, D. (2015) General Basic Science and Pathogenesis. In: Saltzman, B.M., Ed., Heterotopic Ossification: Basic Science, General 
Principles, and Clinical Correlates on Orthopedic Surgery, Nova Science, New York.

[14] Rubin, M.M. and Cozzi, G.M. (1986) Heterotopic Ossification of the Temporomandibular Joint in a Burnt Patient. Journal of Oral and Maxillofacial Surgery, 44, 897-899. https://doi.org/10.1016/0278-2391(86)90228-4

[15] Lindqvist, C., Soderholm, A.L., Hallikainin, D., et al. (1992) Erosion and Heterotopic Bone Formation after Alloplastic Temporomandibular Joint Reconstruction. Journal of Oral and Maxillofacial Surgery, 50, 942-949. https://doi.org/10.1016/0278-2391(92)90051-Z

[16] Durr, E., Turlington, E.G. and Foote, R.L. (1993) Radiation Treatment of Heterotopic Bone Formation in the Temporomandibular Joint Articulation. International Journal of Radiation Oncology, Biology, Physics, 27, 863-869.

https://doi.org/10.1016/0360-3016(93)90461-4

[17] Wolford, L.M. and Karras, S.C. (1997) Autologous Fat Transplantation around Temporomandibular Joint Prostheses: Preliminary Treatment Outcomes. Journal of Oral and Maxillofacial Surgery, 55, 245-251.

https://doi.org/10.1016/S0278-2391(97)90535-8

[18] Reid, R. and Cooke, H. (1999) Postoperative Ionizing Radiation in the Management of Heterotopic Bone Formation in the Temporomandibular Joint. Journal of Oral and Maxillofacial Surgery, 57, 900-905. https://doi.org/10.1016/S0278-2391(99)90003-4

[19] DuVal, M., Davidson, S., Ho, A., et al. (2007) Albright's Hereditary Osteodystrophy with Extensive Heterotopic Ossification of the Oral Maxillofacial Region: How Fetuinresearch May Help a Seemingly Impossible Condition. Journal of the Canadian Dental Association, 73, 845-850.

[20] Mercuri, L.G., Ali, F. and Woolson, R. (2008) Outcomes of Total Alloplastic Replacement with Periarticular Autogenous Fat Grafting for Management of Reankylosis of the Temporomandibular Joint. Journal of Oral and Maxillofacial Surgery, 66, 1794-1803. https://doi.org/10.1016/j.joms.2008.04.004

[21] Pensler, J.M., Christopher, R.D. and Bewyer, D.C. (1933) Correction of Micrognathia with Ankylosis of the Temporomandibular Joint in Childhood. Plastic and Reconstructive Surgery, 91, 799-805. https://doi.org/10.1097/00006534-199304001-00007

[22] Lindquist, C., Pihakari, A., Tasamen, A., et al. (1986) Autogenous Costochondral Grafts in Temporomandibular Joint Arthroplasty: A Surgery of 66 Arthroplasties in 60 Patients. Journal of Maxillofacial Surgery, 14, 143-149. https://doi.org/10.1016/S0301-0503(86)80280-6

[23] Cella, J.P., Salvati, E.A. and Sculco, T.P. (1988) Indomethacin for the Prevention of Heterotopic Ossification Following Total Hip Arthroplasty. The Journal of Arthroplasty, 3, 229-234. https://doi.org/10.1016/S0883-5403(88)80020-2

[24] Jones, P. and Lamdin, R. (2010) Oral Cyclo-Oxygenase 2 Inhibitors versus Other Oral Analgesics for Acute Soft Tissue Injury: Systematic Review and Meta-Analysis. Clinical Drug Investigation, 30, 9-37. https://doi.org/10.2165/11533350-000000000-00000

[25] Jensen, A.W., Viozzi, C.F. and Foote, R.L. (2010) Long-Term Results of Radiation Prophylaxis for Heterotopic Ossification in the Temporomandibular Joint. Journal of Oral and Maxillofacial Surgery, 68, 1100-1105. https://doi.org/10.1016/j.joms.2009.12.018

[26] Popovic, M., Agarwal, A., Zhang, L., et al. (2014) Radiotherapy for the Prophylaxis of Heterotopic Ossification: A Systematic Review and Meta-Analysis of Published 
Data. Radiotherapy and Oncology, 113, 10-17.

https://doi.org/10.1016/j.radonc.2014.08.025

[27] Rosella, D., Papi, P., Giardino, R., et al. (2016) Medication-Related Osteonecrosis of the Jaw: Clinical and Practical Guidelines. Journal of International Society of Preventive and Community Dentistry, 6, 97-104.

https://doi.org/10.4103/2231-0762.178742

[28] Wolford, L.M., Morales-Ryan, C.A., Morales, P.G. and Cassano, D.S. (2008) Autologous Fat Grafts Placed around Temporomandibular Joint Total Joint Prostheses to Prevent Heterotopic Bone Formation. Proceedings (Baylor University. Medical Center, 21, 248-254. https://doi.org/10.1080/08998280.2008.11928404

[29] Shan, Y.Z., Liu, H., Yang, C., Zhang, X., Abdelrehem, A., Zheng, J., Jiao, Z., Chen, M. and Qiu, Y. (2015) Modified Surgical Techniques for Total Alloplastic Temporomandibular Joint Replacement: One Institution's Experience. Journal of Cranio-Maxillofacial Surgery, 43, 934-939. https://doi.org/10.1016/j.jcms.2015.03.028

[30] Selbong, U., Rashidi, R. and Sidebottom, A. (2016) Management of Recurrent Heterotopic Ossification around Total Alloplastic Temporomandibular Joint Replacement. International Journal of Oral and Maxillofacial Surgery, 45, 1234-1236. https://doi.org/10.1016/j.ijom.2016.02.017

[31] Dimitroulis, G. (2004) The Interpositional Dermis-Fat Graft in the Management of Temporomandibularjoint Ankylosis. International Journal of Oral and Maxillofacial Surgery, 3, 755-760. https://doi.org/10.1016/j.ijom.2004.01.012 